\title{
APRENDER DE LO MEJOR: NUEVOS MODELOS PARA UN DESARROLLO Y COOPERACIÓN ADECUADOS
}

\author{
(LEARNING FROM THE BEST: NEW PARADIGMS FOR SUSTAINABLE DEVELOPMENT AND \\ COOPERATION)
}

Nicholas You

Coordinador del Programa de Mejores Prácticas y Liderazgo Local del Centro de las Naciones Unidas para los Asentamientos Humanos(CNUAH)

CHINA

Fecha de recepción: $29-\mathrm{XII}-99$

\section{RESUMEN}

La Conferencia Hábitat II -La Cumbre Urbana-, que tuvo lugar en Estambul (Turquia) en junio de 1996, adoptó la Agenda Hábitat -un Plan de Acción Global para mejorar las condiciones de habitabilidad sostenible en un mundo de rápido desarrollo urbanistico- La Conferencia abrió dos nuevos frentes: (I) fomentar la colaboración entre los gobiernos a todos los niveles y los grupos más relevantes de la sociedad civil; y (2) reconocer la contribución significativa de las ciudades y de la sociedad civil con el fin de poder alcanzar el doble objetivo de vivienda para todos $y$ de sostenibilidad de los asentamientos humanos.

Actualmente las ciudades empiezan a tener tanta importancia o más que las propias naciones en el ámbito del comercio global y las inversiones. Consideradas como centros de producción y consumo son el motor del crecimiento económico y del desarrollo. También puede considerárselas como lugares privilegiados para la educación e investigación, asi como para la posibilidad de poder aplicar nuevas tecnologias, además de ser eje central de la expresión cultural y de la vitalidad social. Sin embargo, las ciudades son tambien un foco de problemas sociales, económicos y de medio ambiente. El crimen, la pobreza, el desempleo, la congestión, la devastación y degradación del entorno natural, son varios de los signos más visibles de estos problemas. La Agenda Hábitat reconoce que los problemas se transforman rápidamente en universales y no pueden ser atajados individualmente y con eficacia por gobiernos, ciudades, comunidades o sectores privados. Requieren, por contra, de la unión de todos con el fin de aprovechar los recursos humanos, tecnológicos y financieros para concebir y aplicar soluciones innovadoras. Asimismo, se requiere un esfuerzo sistemático para intercambiar conocimientos y experiencias, competencias y tecnologias.

\section{SUMMARY}

The habitat II Conference -The City Summit-held in Istambul (Turkey) in june 1996 adopted the Habitat Agenda -a Global Plan of Action to improve living conditions on a sustainable basis for all people in a rapidly urbanising world. The Conference broke new ground on two fronts: (l) in brokering partnerships between governments at all levels and all major groups of civil society; and (2) in recognising the critical contribution of cities and civic society in achieving the twin goals of shelter for all and sustainable human settlements.

Cities are already becoming as important if not more important than nations as front-line actors in global trade and investment. As centres of production and consumption, they are the engines of economic growth and development. They are also centres of excellence in education, research and development, the application of new technologies, the loci of cultural expression and social vitality. Cities, howewer, are also plagued by social, economic and environmental problems. Crime, poverty, unemployment, congestion, waste and the degradation of the natural environment are some of the most visible signs of these problems. The Habitat Agenda recognises that these problems are rapidly becoming universal and that they cannot be effectively addressed by governments, cities. communities or the private sector alone. They require partnerships to harness the human, technical and financial resources to devise and implement innovative solutions. They also require a systemaiic effort for the sharing of knowledge and experiences, expertise and technologies. 


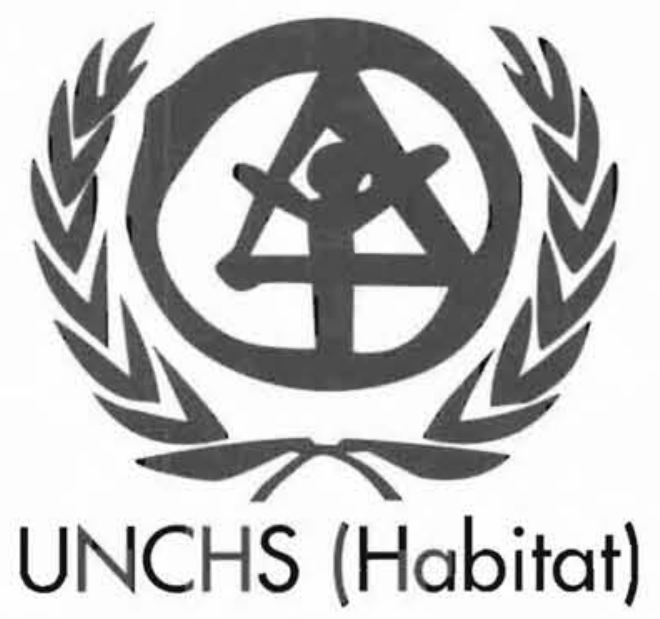

Centro de Naciones Unidas para los Asentamientos Humanos.

\section{INTRODUCCIÓN}

El Centro de las Naciones Unidas para los Asentamientos Humanos (CNUAH) -Hábitat- lanzó una iniciativa globalencaminada a la búsqueda de soluciones efectivas a los problemas relacionados con la sostenibilidad de las condiciones de habitabilidad de las ciudades. Bajo el nombre de Iniciativa de las Mejores Prácticas, durante los años 1995-96 y, a través de dicho organismo, se estudiaron unos 600 casos provenientes de más de 80 países con la finalidad de aunar esfuerzos para mejorar el entorno humano. Las prácticas fueron revisadas por un Comité Técnico Consultivo, el cual seleccionó 104 de dichas prácticas que fueron posteriormente revisadas por un Jurado independiente, entregándose luego en Tokio y Dubai los premios a la mejora del entorno humano, premios éstos que fueron concedidos a doce de los participantes provenientes de diversas ciudades, comunidades y sectores públicos y privados, en reconocimiento a su importante aportación en la resolución de algunos de los problemas más acuciantes de la sociedad, la economía y el medio ambiente, con vistas a la posibilidad de hacer frente a los retosque presentará en el futuro un mundo vertiginosamente cambiante en lo que al urbanismo se refiere.

\section{Qué es lo que define una Mejor Práctica}

La definición de una Mejor Práctica fue adoptada por la Asamblea General de las Naciones Unidas, según los siguientes postulados:

- ASOCIACIÓN: entre dos o más agencias públicas, autoridades locales, organizaciones no gubernamentales y organizaciones de base, sector privado, profesional y académico, etc.;

- IMPACTO: tangible, de las mejoras en el entorno humano;

- SOSTENIBILIDAD: evidenciada por amplios cambios en la Legislación, en la capacidad institucional, en los sistemas de gestión, en los procesos de toma de decisiones y del reparto de recursos y en la gestión financiera y ambiental.

Este proceso se repitió recientemente en 1998 con unas 450 nuevas iniciativas, 127 Mejores Prácticas y la adjudicación de 10 premios de esta categoría.

\section{Premios a las Mejores Prácticas de 1996 y 1998}

Las siguientes iniciativas fueron los primeros premios concedidos a la Mejora del Entorno Humano en la Cumbre de Ciudades en Estambul (1996):

1. Proyecto concerniente a Obras \& Servicios para grupos de bajo nivel económico en North Gran, Buenos Aires, Argentina.

2. Colaboración del Ayuntamiento en el proceso de rehabilitación de las Favelas, en Fortaleza, Brasil.

3. Respuestas innovadoras en Comunidades cambiantes para el Metro de Toronto. Metro de Toronto, Canadá.

4. Reconstrucción de las áreas rurales de la Provincia de Anhui, China.

5. Institucionalización del desarrollo de las Comunidades de base, Comuna de Adjamé, Abidjan, Costa de Marfil.

6. Organización autónoma femenina para paliar la pobreza en la India: el Banco SEWA.

7. Mejora de los asentamientos en Agadir, Marruecos.

8. Gestión Administrativa en Tilburg. "El modelo Tilburg", Holanda.

9. Programa de iniciativa local. Proceso de Planificación Comunitaria y Asociaciones de Barrio, en Lublin, Polonia.

10. Centro de Recursos e Información Comunitaria(CIRC), Alexandra, África del Sur.

11. Enfoque holístico de la sostenibilidad. Ciudad de Chattanooga, Tennessee, EEUU.

12. "Dont' Move Improve", Una Comunidad alienta y dirige un Proyecto de Revitalización Urbana, South Bronx, Nueva York, EEUU.

El Premio Internacional Dubai 1998, a la Mejora del Entorno Humano fue concedido a las siguientes iniciativas:

1. Mejora Global del Entorno Urbano de Zhuhai, China.

2. Sub-centros urbanos para ciudadanos en áreas de escasos recursos económicos, en Medellín, Colombia. 
3. Manejo del desecho sólido casero - Colectores de basura de Zabbaleen, El Cairo, Egipto.

4. Programa de vivienda para las áreas periféricas de Xalapa, Veracruz, Méjico.

5. Gobernabilidadurbana y gestión ambiental y pública en Surat City, India.

6. Proyecto Kipepeo (Mariposa), Arabuko-sokoke Forest, Kenia

7. Iniciativas de planificación participativas en Naga City, Filipinas.

8. Programas para mejorar el Entorno Urbano en Málaga, España.

9. Programa de mejora de la infraestructura Comunitaria, Dar-es Salaam, Tanzania.

10. Jomadas de interrelación de la sostenibilidad, Georgia, EEUU.

\section{Las lecciones aprendidas}

Un análisis de las Mejores Prácticas de 1996 y 1998, reveló diversos factores comunes que contribuyeron al éxito, como por ejemplo:

\section{Asociaciones y participación}

Una de las lecciones más importantes recibidas a través de las Mejores Prácticas fue el nacimiento de un auténtico sistema de asociación -un concepto éste que va más allá de la pura participación-. Entre las prácticas más apremiantes, se encuentra el reconocimiento formal a todos los "stakeholders", independientemente de su habilidad o capacidad, por cuanto a su contribución técnica o financiera se refiere. Ideas, puntos de vista, aportaciones en especie -aunque pequeñas- son igualmente valoradas, tanto como la de aquellos socios que aportan, a este esfuerzo, una financiación sustancial o un compromiso social. En Fortaleza (Brasil) -una de las Mejores Prácticas ganadora de 1996-, se da el ejemplo de un vertedero de basura inundado de agua, que se transformó, posteriormente, en un núcleo de viviendas y zona de pequeños negocios, graciasa la asociación con las autoridades locales, cuidando tanto los aspectos materiales de la rehabilitación como de la integración poblacional, en lo que se refiere a una planificada utilización del suelo y a la provisión y mejora de los servicios sociales e infraestructura básica. En la ciudad de Tilburg (Holanda), se concedió otro premio de Mejores Prácticas a un cambio en la mentalidad del gobierno en su nueva visión sobre los ciudadanos ya que éstos fueron considerados como accionistas dentro de una empresa urbana, cuyos beneficios se midieron en términos de calidad de servicio y nivel de transparencia y responsabilidad.

\section{Participación comunitario}

Los premios de Mejores Prácticas del año 1998 permitieron demostrar lo importante que resulta la participación de la comunidad en la mejora del entorno humano. La evidencia de esta participación quedó de manifiesto en diversas Mejores Prácticas donde personas con un nivel bajo de ingresos y vivienda en asentamientos ilegales, tuvieron acceso al mercado de trabajo, a albergues, tierras y servicios básicos. Un ejemplo de ello se produjoen Dar-es-Salaam (Tanzania), en un programa de Infraestructura Comunitaria. Las autoridades locales, conscientes de la imposibilidad de asumir todas las demandas de los residentes en cuanto a servicios básicos se refiere, puso en marcha un Programa de Infraestructura Comunitaria (para mejora de la calidad), con el fin de ayudar a las comunidades a mejorar sus propios asentamientos. El programa, iniciado en 1995, se desarrolló en contacto directo con las comunidades, con la finalidad de intensificar tanto la organización como la ejecución y planificación de las actividades monitoras, y con el propio Ayuntamiento, para mejorar las posibilidades de trabajo con las comunidades, con el fin de ejecutar conjuntamente proyectos de infraestructura. En Tabata, un suburbio con población de bajo nivel económico, se está desarrollando un sistema para el suministro de agua, pagado y realizado por los propios residentes, cuyos beneficios serán destinados, en compensación, a colaborar financieramente en la regeneración de los eriales. La Comunidad contribuirá con un $5 \%$ del coste total de la infraestructura de conducciones, además de la negociación de una red de carreteras más importante que la inicialmente realizada por el Banco Mundial. El alto sentido comunitario y de responsabilidad ayuda a asegurar el éxito del Programa en el futuro.

Igualmente importantes son aquellos casos donde la población fue autorizada y estimulada para tomar el asunto en sus manos, con el fin de adquirir un sentido de propiedad y control de aquellas decisiones que afectan al medio ambiente en su trabajo. En 1994, Ray C. Anderson, el CEO y Presidente de Interface Inc., una importante empresa de fabricación de alfombras, decidió convertir Interface en una empresa de "restauración", apoyando a los empleados en todas las operaciones. Hoy en día, con una plantilla de 7.400 personas, los directores son responsables de realizar el mantenimiento con sus propias unidades y de compartir las Mejores Prácticas y recursos con otras unidades. Su implicación activa ha conducido hacia un mayor desarrollo de la creatividad y a la ejecución de nuevas ideas en distintos frentes: eliminación de los residuos y emisiones benignas; utilización de energia renovable; cierre de los ciclos de vida de los materiales; transportes más eficaces; nuevo diseño de marketing y servicios de entrega, así como hacer que la comunidad sea más consciente de la necesidad de proteger al medio ambiente. El programa se extendió asimismo a las asociaciones, suministradores y comerciantes, a través de 
iniciativas tales como greening the supply chain, formando una red con todos ellos a través de asociaciones comerciales, conferencias y organizaciones de apoyo. Desde 1994, la compañia pudo ahorrar aproximadamente unos 50.000.000 de \$ USA y el precio de existencias en almacén se ha cuadruplicado.

\section{Toma de decisiones por parte de la Comunidad}

Una de las características más destacadas de muchas de las Mejores Prácticas es la gran participación de la comunidad en problemas de identificación, planificación, diseño de los proyectos y ejecución de los mismos. Un caso muy destacable tuvo lugar en Chattanoga, Tennessee (EE UU), durante los premios Mejores Prácticas de 1996, pues el proceso inicial de toma de decisiones para solucionar un problema de polución del aire se extendió a lo largo de 10 años y llevó consigo la solución de un sinfin de problemas muy complejos, entre los cuales se encuentra el cambio de política y estrategias y la inversión de un capital superior a los 700.000 .000 de $\$$ USA provenientes de muy diversos lugares, tanto privados como comunitarios. Desde ese momento, Chattanooga fue reconocida como una de las ciudades más limpias de los Estados Unidos, no sólo por el hecho de haber solucionado el problema de la polución, sino también por haber creado una serie de iniciativas, como por ejemplo la construcción de viviendas para grupos de bajos ingresos, la revitalización del centro de la ciudad -con la integración de un parque y una red pública de autobuses eléctricos-, amplio reciclado de desechos $\mathrm{y}$, en resumen, la eliminación de todo punto contaminante.

\section{Posibilidad de construir a través de la formalización y consolidación participativas}

Aunque muchas de las Mejores Prácticas se iniciaron como proyectos, aquéllas que provienen del autoabastecimiento muestran que el aprendizaje de la especialidad de transformación y la destreza son tan importantes como la técnica en sí misma y la experiencia. El aprendizaje del oficio incluye estar en posesión de dotes de mando, disponer de equipos de construcción, poder resolver conflictos y negociaciones, participar en la planificación, movilizar a la comunidad y gestionar la organización e información (producción, utilización y diseminación). La participación de muy diversos stakeholders y socios en estos procesos sistemáticos, constituye un ejercicio de capacitación para la construcción de comunidades enteras, además de asegurar que el aprendizaje de dichas especialidades puede ser aplicado continuamente más allá de la planificación inicial y la ejecución de las diferentes etapas, a la gestión administrativa del proceso de desarrollo local. En muchos casos estas competencias permitieron el mejor uso e integración de los expertos técnicos y profesionales, dentro de una continua gestión y puesta en práctica. Una de

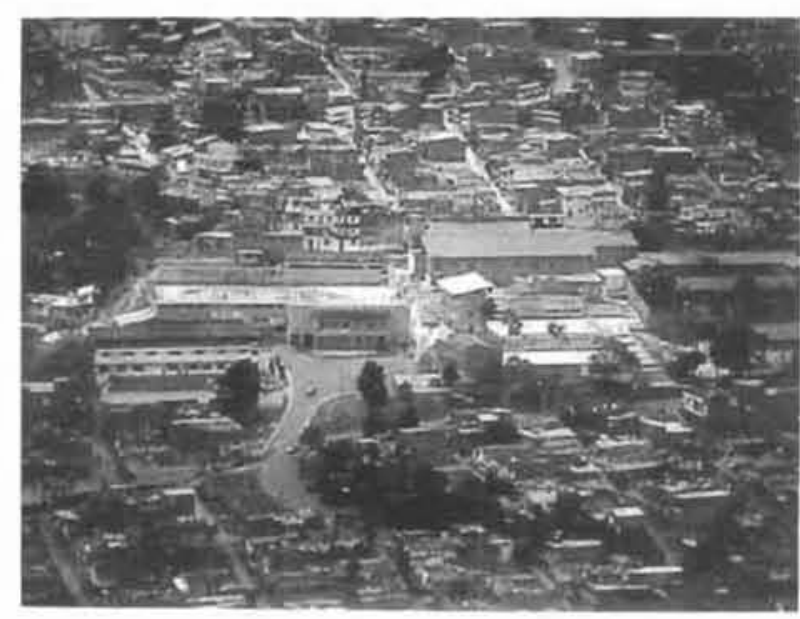

Subcentros urbanos para ciudadanos en áreas de escasos recursos económicos, Medellin, Colombia.

las lecciones clave aprendidas a través de diversas Mejores Prácticas, es la necesidad de fortalecer y formalizar los procesos de toma de decisión y participación. Además, la necesidad de tal fortalecimiento es igualmente evidente en el ámbito de ciudad, como en Naga City (Filipinas), o en el caso del desarrollo de un problema de índole privado en Medellín (Colombia).

En 1991, en las Islas Filipinas, el Código de las Autoridades Locales pidió a los regidores locales que consultaran al pueblo para hacerle asi participar en la planificación del desarrollo local. Sin embargo, pronto se dieron cuenta de que no tenían capacidad suficiente para participar en el programa. En diciembre de 1995 se decretó la Ordenanza de poderes de 1996, la cual reconocía oficialmente ciertas entidades encargadas de la población rural, bajo el patrocinio del Consejo de la Población de la Ciudad de Naga $(\mathrm{CPCN})$, como legítimos actuantes en la planificación del desarrollo local. La Ordenanza institucionalizaba la participación del pueblo en la planificación y gestión urbana, facilitando además la participación en el ámbito laboral tanto en el sector público-privado, como en el comunitario. En una segunda fase, se creó la iniciativa de planificación participativa de la Ciudad de Naga. Como resultado de la misma, se aumentó ampliamente la participación del pueblo en el desarrollo local, incrementando el sentido de copropiedad en los ciudadanos en cuanto a los proyectos y programas urbanísticos se refiere.

Medellín (Colombia) está considerada como una de las ciudades más duras del mundo donde el crimen y la violencia urbana se han cuadruplicado en la última década debido, sobre todo, al aumento del tráfico de drogas, al crecimiento descontrolado de la demografía y al estancamiento económico. En 1990, se estableció un amplio plan para frenar la inseguridad crítica creada por el narcotráfico, involucrando en el mismo al gobierno central, al Programa de las Naciones Unidas para el Desarrollo(PNUD), organizaciones no gubernamentales, 
investigadores y presidentes de comunidades. La iniciativa se llevó a cabo a través de un modelo de comunidad, con participación pública en la administración local. Se desarrolló el sistema educativo en varios suburbios de bajo nivel económico, así como el sistema sanitario, nutricional y la participación cívica. A través de estos subcentros, los residentes trabajaron conjuntamente con el gobierno, las autoridades locales y el sector privado con el fin de mejorar sus comunidades y barrios.

\section{Transparencia, confianza y franqueza}

Una vez establecidos los procesos participativos para un proyecto específico o iniciativa, la necesidad de mantener y alimentar dichos procesos se perfiló como un desafio en aumento que necesitaba de transparencia, confianza y franqueza. El resultado fue muy alentador, ya que el mismo proceso pudo ser aplicado para resolver otros problemas, con frecuencia más complejos y quizás más importantes, con el fin de paliar los frecuentes cambios políticos.

En las afueras de Xalapa (Méjico), en el Estado de Veracruz, una comunidad de bajo nivel económico negoció con éxito con las autoridades del Estado y la ciudad, la posibilidad de mejorar el entorno. En 1991, una organización comunal llamada Unión de Arrendatarios y Postulantes, desarrolló un Plan para 80 barrios con habitantes de bajos ingresos en la periferia de Xalapa, plan que fue aprobado tanto por el Estado como por las autoridades locales. Dicho plan consistía en integrar a la comunidad en el ámbito de la ciudad, para que fuera reconocida como parte de ella. Para llevarlo a cabo, la comunidad recibió ayuda de la Fundación Ford y de NOVIB -una ONG holandesa-, recibiendo información sobre la forma de resolver los problemas de planificación y vivienda. Puesta en marcha esta iniciativa con el respaldo del apoyo oficial, en 1997 CENVI, una ONG mejicana, puso en marcha un Plan de Mejora Urbana e
Integración Social, que incluía un proyecto de viviendas, un programa de créditos a las mujeres y proyectos de educación y nutrición en la zona, con el consiguiente fortalecimiento de la participación directa del pueblo y de las comunidades en la toma de decisiones sobre la forma y significado de la mejora de su calidad de vida.

En El Cairo (Egipto), los Zabbaleen (colectores de basura) no sólo venden productos fabricados a partir de la basura reciclada, sino que el hecho en sí del reciclado produce una influencia positiva en la política del gobierno por cuanto se refiere al pobre urbano. En los Zabbaleen se colectan y reciclan aproximadamente unas 600 toneladas de desechos domésticos por día, es decir, más o menos un tercio de los detritus producidos por los residentes de la ciudad. Los ingresos generados por estas actividades de reciclado se invirtieron en la construcción de casas, infraestructuras y servicios básicos, contribuyendo ampliamente a la mejora de la salud y del bienestar de la comunidad. El esfuerzo conjunto del sector privado y de las ONGs, es un excelente ejemplo de que el tratamiento de los desechos sólidos puede no sólo convertirse en una fuente de ingresos para grupos de bajos recursos, sino que también contribuye a mejorar el entorno urbano de la zona más pobre de la ciudad. Este colectivo está involucrado ahora en una nueva fase de expansión que incluye la separación de los desechos por los residentes del barrio y la construcción y puesta en marcha de un nuevo complejo industrial dedicado al reciclaje.

\section{Planificación Estratégica y Comprensible}

La Agenda Hábitat y la Agenda 21 reconocen claramente la necesidad de dirigir los problemas sociales, económicos y de medio ambiente de forma holistica. Esto requiere la búsqueda de políticas y estrategias visionarias basadas en las posibilidades de progreso y efectividad a lo largo del camino. Tanto Málaga (España) como Zhuhai (China) trabajan en la conservación de su herencia cultural

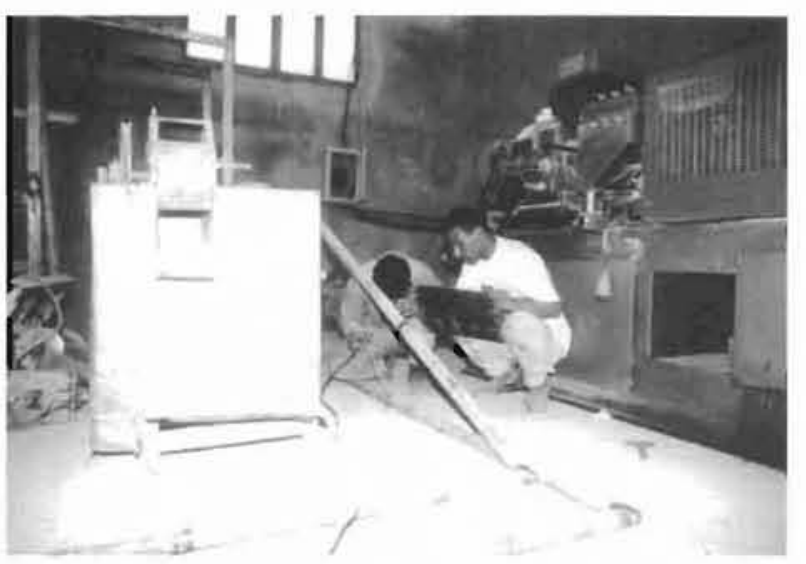




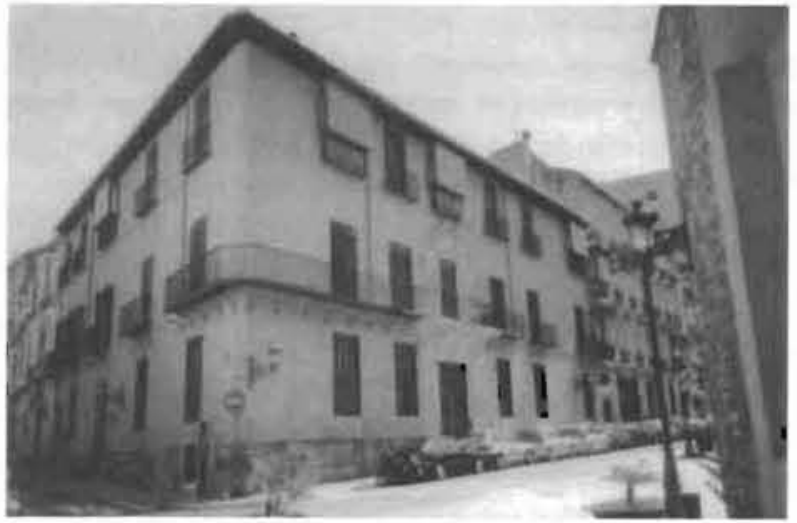

Programas para mejorar el Entorno Urbano. Rehabilitación de fachadas. Málaga, España.

e histórica enfocando sus solicitudes de ayuda hacia una rápida expansión y la solución del ruido ambiental.

Durante los últimos 20 años, la ciudad de Málaga (España) ha experimentado un rápido crecimiento en su industria turistica, aumentando su población hasta, aproximadamente, medio millón más de personas, lo que contribuyó al deterioro de su infraestructura urbana y su entorno. En 1995, el Ayuntamiento aprobó el Estatuto para una Málaga Verde, enfocado hacia la rehabilitación del centro histórico, con la aportación de servicios de recuperación ambiental en el área metropolitana, contribuyendo así a convertir una zona contaminada en un parque frente al mar. Como consecuencia de la toma de esta decisión, se restauraron, además, varios edificios históricos, se adecuaron espacios públicos, nuevos parques e incluso se construyó una gran planta de tratamiento de agua, al mismo tiempo que se recuperaba una de las playas. También se instalaron monitores donde podía verse el desarrollo del plan. Estas actuaciones son una muestra de cómo la Agenda 21 y la Agenda Hábitat pueden elevar el nivel local.

En cierta ocasión un pobre pueblo pesquero, Zhuhai, en la provincia de Guangdong, al sur de China, se transformó en una ciudad modelo gracias a una eficaz planificación urbana y a una buena concepción del medio ambiente. En 1980, el ayuntamiento de Zhuhai construyó un nuevo distrito urbano de 56,2 kilómetros cuadrados, que contribuyó a mejorar la infraestructura de la ciudad, expandiendo su espacio para un futuro desarrollo, mejorando asimismo la calidad del aire y del agua. El éxito de esta iniciativa -que dobló el "Proyecto primitivo"- no sólo recibió los honores de la ciudad, sino que también animó a otras ciudades a repetir los esfuerzos de Zhuhai en la renovación urbanística y la recuperación del medio ambiente.

\section{Liderazgo y cambio}

Cada Mejor Práctica se ha enfrentado con demasiada

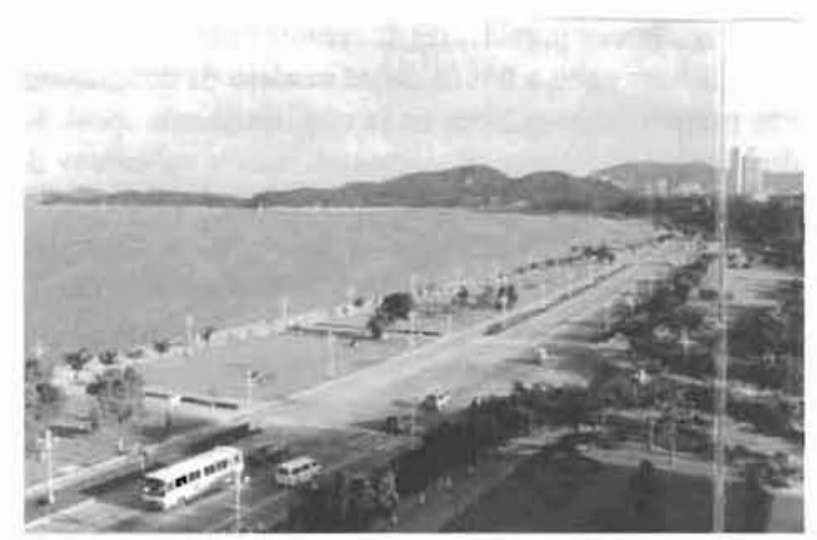

Mejora global del Entorno Urbano de Zhuhai. China

frecuencia a otro tipo de prácticas no tan buenas, calificadas como "práctica aceptable" o "business as usual". El liderazgo, con lo que lleva consigo en lo que se refiere al valor de adelantarse a todos en los cambios de actitud yen los procedimientos operativos y estándares de conducta, ha dejado bien claro que se trata de un aspecto esencial en el momento de llevar a cabo el cambio.

El Ayuntamiento de Surat(India) fue siempre considerado como una de las municipalidades más moribundas de la India. Su nula posibilidad de salir adelante quedó bien patente en septiembre de 1994 cuando se desató una plaga que ni la ciudad ni la municipalidad fueron capaces de contener. Por entonces, tan sólo un 45 por ciento de los residentes tenían acceso al agua y a los servicios sanitarios y el mantenimiento de las conducciones para desagües, alumbrado, parques, carreteras y escuelas eran totalmente deficientes. En mayo de 1995, el Ayuntamiento puso en marcha un plan participativo y comprensivo, plan que incluía 47 proyectos enfocados hacia los sistemas de carreteras, dirección del tráfico, suministro de agua, depuración de aguas residuales, drenaje del agua de las tormentas, gas, electricidad, mejora de los barrios bajos, etc.; mejorar la organización, fomentar la opción a nuevas posibilidades, asegurar la transparencia y crear una concienciación pública. En un plazo de 18 meses, Surat fue considerada como la segunda ciudad más limpia de la India. Poco antes de recibir, en 1998 el Premio Internacional Dubai, la ciudad de Surat fue puesta a prueba de nuevo con graves inundaciones. En esa ocasión no se produjeron epidemias y la vida volvió a su cauce tan sólo una semana después.

Con frecuencia, este papel de lider es asumido por un agente de cambio foráneo o por una decisión transcendental como es el caso del proyecto Kipepeo (Mariposa), en Kenia. El Bosque "Arabuko-Sokoke", situado en la costa norte de Kenia, es una isla de biodiversidad semi-urbana, amenazada tanto por una agricultura de subsistencia como por un turismo en desarrollo. Contiene seis especies de 

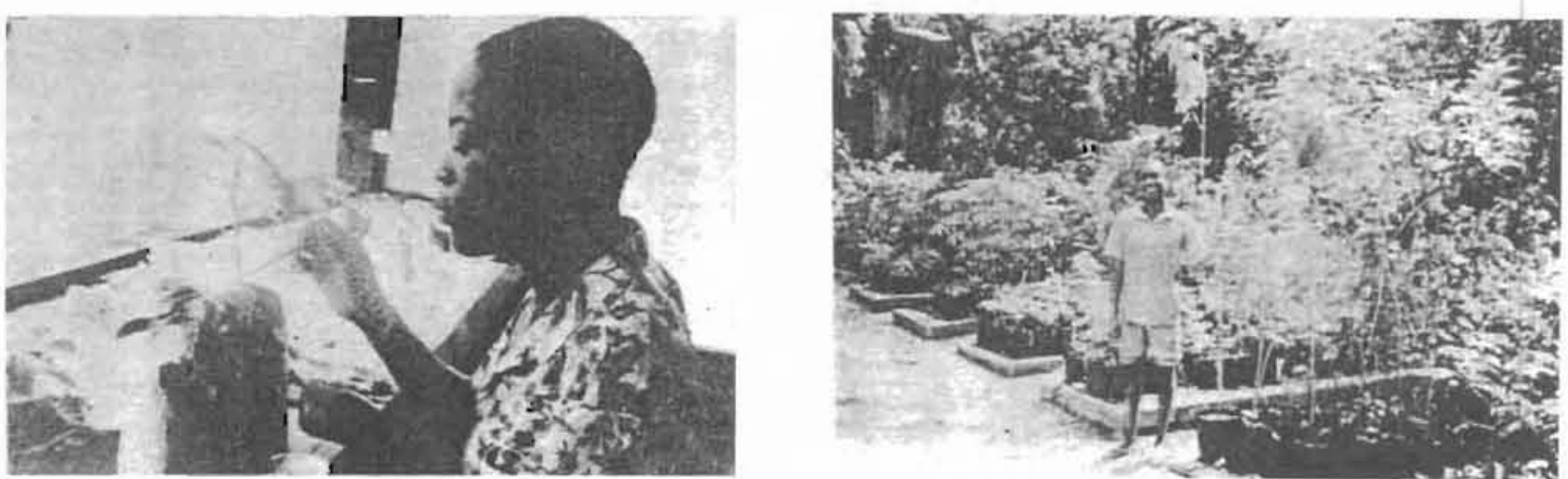

Proyecto Kipepeo (Mariposa), Arabuko-Sokoke Forest, Kenia.

aves amenazadas (siendo el segundo bosque de mayor importancia de Africa por lo que concierne a la conservación de aves), cuatro especies amenazadas de mamíferos y un número desconocido de otras especies, rodeado de granjeros cuyos ingresosper capita no pasaban de los 50 \$ USA. En 1991 se realizó una encuesta acerca del bosque, que puso en evidencia que el 96 por ciento de los encuestados deseaban deshacerse de él, debido a las incursiones de los animales salvajes en los campos y de la necesidad de más tierra de cultivo. Tal acontecimiento hubiera sido considerado como algo normal en una región donde la naturaleza y la conservación de los animales salvajes están a expensas de una población en rápido crecimiento que debe procurarse su propio sustento. El proyecto Kipepeo planteó el desafío de demostrar el hecho incontestable de que, manteniendo la biodiversidad, no sólo se beneficiaba la comunidad sino, que también constituía un modelo viable de coexistencia entre el medio rural y el urbano. El desarrollo del proyecto llevó consigo la formación de 150 granjeros dedicados a la crianza de mariposas de bosque utilizando las hojas de los árboles. La mariposa pupae se exporta tanto a Europa como a América del Norte. Desde 1994, Kipepeo consiguió para Kenia cerca de 100.000 SUSA en divisas, habiendo pagado aproximadamente unos 35.000 a los granjeros. Una nueva encuesta realizada a éstos en 1998, mostró un mayor deseo conservacionista, ya que el 84 por ciento de ellos se mostraban de acuerdo con la conservación del bosque. La formación de los granjeros no fue adversa por cuanto se refiere a la conservación de las mariposas autóctonas.

\section{Transferencia de Conocimientos, Competencias y Experiencia}

Un resultado inesperado por lo que se refiere al seguimiento de los trabajos tuvo lugar durante los premios de Mejores Prácticas del año 1996, en lo relacionado con las transferencias espontáneas entre comunidades, ciudades y agencias. Entre los doce premios de Mejores Prácticas de 1996, once están, aún hoy en día, activamente dispuestos a aprender los unos de los otros y/o a ayudar a otros grupos a través del intercambio y de la transferencia de sus conocimientos, competencias y experiencia. Dichas transferencias tienen lugar con criterios sur-sur, nortesur y sur-norte. Como ejemplo se puede mencionar a Fortaleza (Brasil) y a otras once municipalidades en Brasil, con Johannesburg (Sudáfrica) y Dunquerque (Francia); el Banco SEWA (India), con la Corporación de Desarrollo Comunitario en Canadá y una operación de préstamo (micro-crédito) en Sri Lanka; y Banana Kelly en Nueva York, con South-Central en Los Angeles.

Estas transferencias y otras que están llevándose a cabo en Europa y Asia, muestran la efectividad de la enseñanza interna y de las formas descentralizadas de cooperación. Lo que realmente se requiere para acceder al desafio global para un desarrollo más sostenible es un sistema donde la experiencia práctica pueda realmente ser accesible a todos aquellos que la requieran, desde la comunidad local a la nacional, asi como a niveles regionales.

\section{Programa de Mejores Prácticas y Liderazgo Local}

La demanda de información de las Mejores Prácticas llevó al CNUAH -Hábitat- a institucionalizar el Programa de Mejores Prácticas y Liderazgo Local (PMPL). Este tipo de programas, junto con los Programas de Indicadores Urbanos y Observatorio Urbano Global tienen como fin alentar el progreso a través de la aplicación de la Agenda Hábitat, el trabajo de una red global y de observatorios regionales, nacionales y locales dentro del ámbito urbano. El PMLP es considerado como un programa de conexión, que utiliza sus propias ordenanzas, dedicado a compartir, intercambiar y transferir los conocimientos de las Mejores Prácticas, competencias y experiencia. Todo ello a través de una red global de centros relacionados con el control en la edificación, asociaciones profesionales, cobertura de las ONGs y asociaciones y autoridades locales. Desde sus comienzos, en 1997, más de 20 instituciones y organizaciones han formado el embrión de este nuevo 

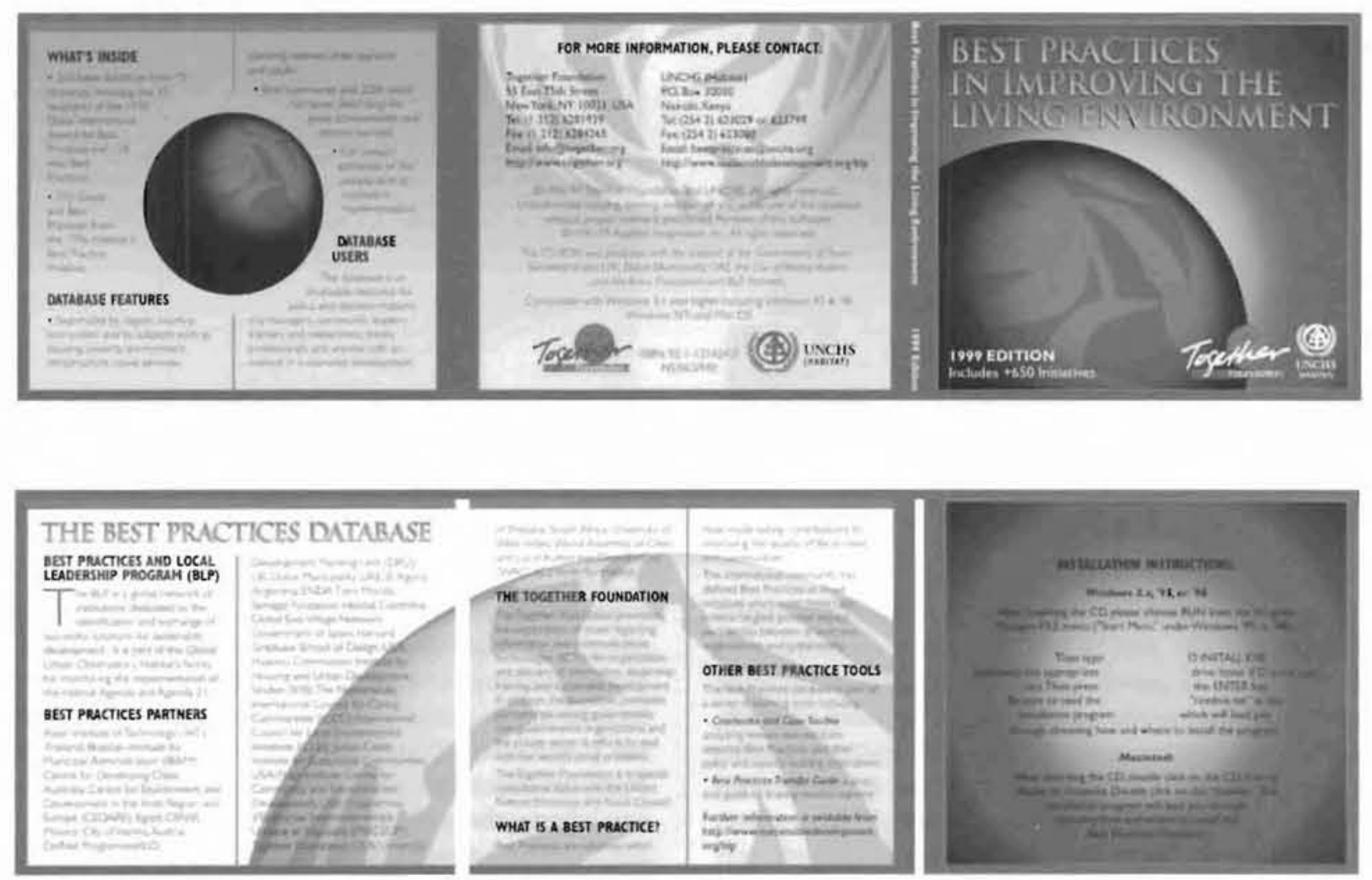

Base de datos de las Mejores Prácticas. Fundación Together.

programa que producirá y mantendrá una serie de productos y servicios para facilitar el equilibrio entre oferta y demanda para un mayor conocimiento, competencias y experiencia de las Mejores Prácticas. Dichos productos y servicios incluyen:

-EI Premio Internacional Dubai de Mejores Prácticas para la Mejora del Entorno Humano. Considerando la importancia de tener una visión global y un sistema de incentivos para ciudades y comunidades que sometan sus iniciativas a consideración, el Ayuntamiento de Dubai, a partir de 1998 y para años sucesivos, decidió donar 10 premios de 30.000 \$ USA cada uno a la Excelencia en la Mejora del Entomo Humano. El plazo de entrega para el año 2000 termina el 30 de abril y las solicitudes pueden enviarse a través de Internet o por disquete. Las solicitudes que lleguen dentro del plazo previsto serán estudiadas por un representante regional independiente y un comité de expertos, cuyas recomendaciones pasarán a un jurado internacional independiente.

- La base de datos de las Mejores Prácticas, herramienta accesible en Internet que permite la búsqueda por paises, regiones, ecosistemas/ecorregiones, escala de intervención, temas o sectores, impulsores de la práctica y palabras clave. Dicha herramienta está diseñada para permitir a planificadores, gestores urbanos o líderes de la comunidad identificar rápidamente iniciativas que se han desarrollado con éxito en otras zonas del mundo y contactar con las personas u organizaciones directamente responsables de su implementación. La base de datos está disponible en CD-ROM, disquetes y en Internet (http:// www.bestpractices.org).

-El sistema de Intercambio y Comunicación Intranet de Mejores Prácticas. Intranet es un sistema con base Internet que permite a los usuarios del Foro Iberoamericano de Mejores Prácticas obtener información, participar de conferencias electrónicas, compartir herramientas de trabajo y métodos aplicados a las iniciativas locales, nacionales y regionales de Mejores Prácticas.

-Una serie de libros, bajo el título de "Mejora de la Gestión y Gobernabilidad Urbana: aprendiendo de las Mejores Prácticas para la Mejora del Entorno Humano". Esta colección de libros que empezó a crearse en 1999 como primer eslabón de la cadena, será de gran ayuda para profesionales, maestros y educadores, dándoles la posibilidad de acceder al análisis de las mejores prácticas en áreas criticas como las de desarrollo económico y generación de empleo, participación pública y privada en la provisión de infraestructura básica y servicios básicos, 
prevención del crimen y justicia social, transporte y movilización y protección del medio ambiente, igualdad genérica e inclusión social.

\section{- Seminarios, talleres, cursos de formación y} conferencias, patrocinados por miembros de la PMPL y por el Foro Iberoamericano y del Caribe, donde la experiencia y el conocimiento de las Mejores Prácticas están siendo ampliamente difundidos entre los principales grupos de socios para su análisis y discusión.

Basándose en la monitorización y la evaluación sistemáticas, el Programa de Mejores Prácticas y Liderazgo Local tiene como finalidad el desarrollo en colaboración con las asociaciones, autoridades locales, asociaciones profesionales, organizaciones no gubernamentales, sector privado e instituciones con capacidad de desarrollo a escala mundial, de una serie de pautas, métodos y herramientas para ser utilizados por el pueblo y sus comunidades, políticas a seguir en la toma de decisiones sobre cómo casar demanda con oferta para obtener un resultado más práctico, competencia y experiencia para que nuestras ciudades y comunidades sean más sanas, seguras, sostenibles y equitativas.

\section{Red de Miembros de las Mejores Prácticas}

El PMLP es una red global descentralizada, integrada entre otros por los siguientes miembros:

- El Instituto de Tecnología de Asia, Bangkok, Tailandia. - Instituto Brasileño de Administración Municipal, Río de Janeiro, Brasil.

- Centro Ambiental y Desarrollo, para la Región Árabe y Europea, El Cairo, Egipto.

- Centro para las Ciudades en vías de Desarrollo (CCD),

Universidad de Canberra, Australia.

- Unidad de Planificación Urbana (UPU), University

College of London, Reino Unido.

- Municipalidad de Dubai, Dubai, EAU.

- Medio Ambiente y Urbanismo(ENDA), Dakar, Senegal.

- Future Base - Ciudad de Viena.

- Global Eco-Village Network (GEN).
- Gobierno de España.

- Universidad de Harvard, Cambridge, MA, EE UU.

- Foro Iberoamericano y del Caribe de Mejores Prácticas (cinco instituciones).

- Instituto de Estudios de Vivienda y Desarrollo (HIS), Rotterdam, Holanda.

- Consejo Internacional de Iniciativas Locales Ambientales, (ICLEI), Toronto, Canadá.

- Instituto Joslyn Castle de Comunidades Sostenibles, Universidad de Nebraska, EE UU.

- Fundación Together, Nueva York, EEUU.

- Universidad de Pretoria, Escuela de Administración Pública \& Gestión, Pretoria, Sudáfrica.

- Coordinación de la Asamblea Mundial de las Ciudades y Autoridades Locales, Ginebra, Suiza.

\section{Algunos datos como conclusión}

La ambición de conseguir un mayor desarrollo durante el siglo XXI va indefectiblemente unida al conocimiento de! valor añadido, información y experiencia. Mientras que las ciudades de un mundo en continuo desarrollo urbanístico seguirán compitiendo con las demás en cuanto a oportunidades de inversión, turismo y comercio se refiere, también habrán de cooperar entre si e internacionalmente, olvidando las fronteras. Asimismo necesitarán aunar experiencia y competencia para que las ciudades sean más habitables. Necesitarán también compartir sus conocimientos y su saber hacer para que sus comunidades sean llevaderas. Sus territorios, roles y responsabilidades necesitarán, para su expansión, abarcar asuntos y problemas que hasta este momento habian sido encomendados a los gobiemos centrales. Estos asuntos $y$ problemas incluyen sanidad, medio ambiente no contaminado, educación, energia, desechos, transporte y comunicación, desarrolloeconómico y creación de puestos de trabajo. También necesitarán mejorar su gobernabilidad para que las energías, los recursos e ideas surgidos de sectores privados y oficiales o de cualquier persona puedan ser movilizados para conseguir una mayor calidad de vida. Finalmente, es la calidad de vida lo que hará que la ciudad sea más o menos atractiva para cuantos en ella trabajan y viven, así como para los negocios e inversiones. 